\title{
GEOTHERMAL HEAT EXTRACTION BY WATER CIRCULATION THROUGH A LARGE CRACK IN DRY HOT ROCK MASS
}

\author{
ZDENĚK P. BAŽANT* AND HIDEOMI OHTSUBO $\ddagger$ \\ Northwestern University, Evanston, Illinois 60201, U.S.A.
}

\begin{abstract}
SUMMARY
One proposed geothermal heat extraction scheme relies on water circulation in a large vertical crack created by hydraulic fracturing in a hot dry impermeable rock mass. Water flow, heat convection and crack opening widths are analysed by finite elements. Governing field equations of the problem are first set up rigorously and then various small terms are identified and neglected, retaining the effects of pressure gradient, buoyancy, velocity head (kinetic energy) and head loss due to viscous friction in the water flow equation, and the effects of heat convection in water and heat conduction in rock in the heat transfer equation. The finite element scheme for water flow is based on a variational principle that is typical for diffusion problems, and for heat transfer it is based on the method of least-square residuals. The system of differential equations is highly non-linear. The non-linear terms and coefficients are treated in the finite element analysis as constant; the finite element analysis of the steady-state pressures, fluxes and temperatures is then iterated, evaluating all non-linear terms and coefficients on the basis of the solution obtained in the previous iteration. Numerically calculated fields at various times after the start of cooling are presented. They indicate some features favourable for the geothermal scheme, such as formation of eddy currents, and downward flux of water toward hotter rock. However, other important questions would have to be solved to gain full understanding of this proposed geothermal scheme.
\end{abstract}

\section{INTRODUCTION}

In one proposed scheme of heat extraction from hot dry rock geothermal reservoirs, a large vertical crack is to be induced by hydraulic fracturing from a borehole (Figure 1). The upper part of the crack is then intersected by a second borehole, and heat is extracted by circulating water through the crack. Influx of cold water is expected to cause secondary fracturing and large expansion of the region from which the heat can be withdrawn.

Considerable interest has been recently shown in this scheme and various aspects of the problem have been studied. ${ }^{1-3}$ One problem of particular interest is the circulation of water, heat convection and opening width of a single, main crack created by hydraulic fracture. This problem was analysed by McFarland ${ }^{4}$ using a standard finite difference program for diffusion flows. The present paper deals with finite element analysis of the problem. Variations of the crack thickness due to temperature changes are taken into account, and differential equations describing the flow and heat convection in the crack more accurately than those used before are developed. These equations are essentially the same as those in a previous preprint ${ }^{5}$ and

\footnotetext{
* Professor of Civil Engineering.

\# Visiting Scholar, Associate Professor on leave from Dept. of Naval Architecture University of Tokyo, Bunkyo-Ku, Tokyo.
}

0363-9061/78/0402-0317\$01.00

(C) 1978 by John Wiley \& Sons, Ltd. 


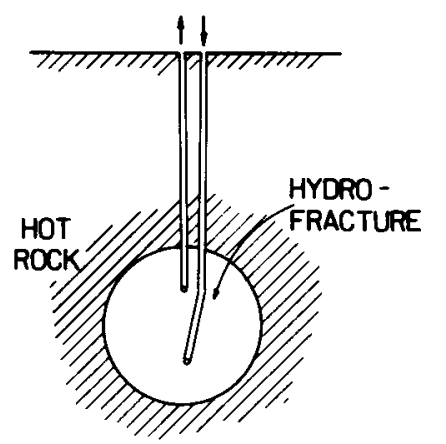

Figure 1. Vertical crack in hot rock

report $^{6}$ where they were derived by integrating the differential equations over the crack width, while here the integrated equations are obtained directly from elementary principles. Similar flow problems have been analysed by finite elements with reference to natural (wet) geothermal reservoirs. ${ }^{7-9}$ An approximate finite difference analysis of water flow through a system of cooling cracks created in hot dry rock has also been made. ${ }^{10}$

\section{GOVERNING EQUATIONS}

Consider a water-filled planar crack whose middle surface coincides with the plane $(x y)$ of Cartesian coordinates $x=x_{1}, y=x_{2}$ and $z$ (Figure 2). The thickness, $w$, of the crack depends on $x$ and $y$, as well as time $t$. The width of the crack is assumed to be negligible compared with
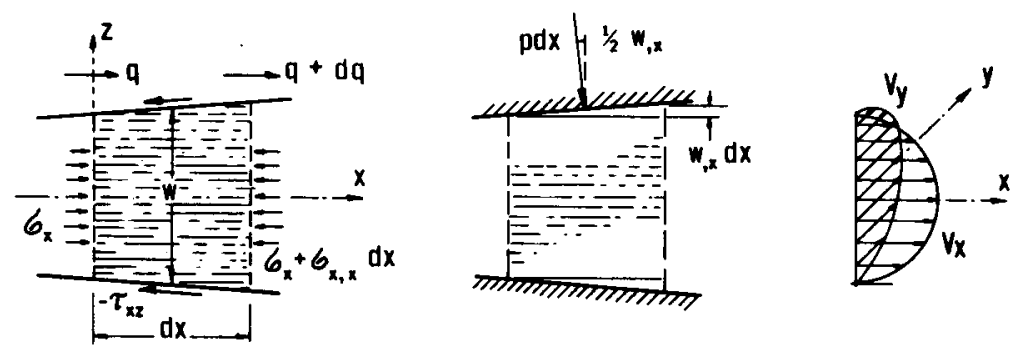

Figure 2. An element of the crack and velocity profile

its length, and so the flow may be characterized integrally for the whole cross-section, taking the variation of $w$ into account. The appropriate equations governing the flow of water and heat transfer in the crack will first be formulated, based on the well-known elementary laws governing the flow of liquids and energy transfer. ${ }^{11}$ For this purpose, pressure $p$, temperature $T$ and mass density $\rho$ may be assumed to be constant throughout the thickness of the crack, while the distribution of velocity vector $\mathbf{v}$ must be considered to be non-uniform, although fixed.

\section{Balance of mass}

The rate of change of the mass of water within an element $\mathrm{d} x \mathrm{~d} y$ of the crack is $\left(\int_{w} \rho \mathrm{d} z\right)^{*} \mathrm{~d} x \mathrm{~d} y$, where the superimposed dot denotes the partial time derivative $\partial / \partial t$ and $\int_{w}$ 
denotes integration from $z=-w / 2$ to $z=w / 2$. The rate of mass flow into the element is $-\left(\int_{w} \rho v_{k} \mathrm{~d} z\right)_{, k} \mathrm{~d} x \mathrm{~d} y$, where a subscript that is preceded by a comma denotes a partial derivative and $v_{k}$ are the components of the vector $\mathrm{v}$ of velocity of water; subscript $k$ refers to $x_{1}=x$ and $x_{2}=y$, and repeated subscripts imply summation over $k=1,2$ (not $k=1,2,3$ ). Conservation of mass requires that both preceding expressions be equal, which yields

$$
(w \rho)^{*}+q_{k, k}=0
$$

in which

$$
q_{k}=w \rho \bar{v}_{k}=\int_{w} \rho v_{k} \mathrm{~d} z \quad(k=1,2)
$$

$q_{k}=$ components of the vector $\mathbf{q}$ of the total mass flux in the cross-section and $\bar{v}_{k}=$ components of the vector $\overrightarrow{\mathbf{v}}$ of average velocity (superimposed bar denotes the average).

\section{Balance of linear momentum}

The vector $\left(\rho v_{1}, \rho v_{2}\right)$ represents the linear momentum, and its total flux within the crosssection is $\int_{w}\left(\rho v_{i}\right) \mathrm{v} \mathrm{d} z(i=1,2)$. The rate of change of linear momentum components within element $\mathrm{d} x \mathrm{~d} y$ of the crack must equal the rate of flow of the linear momentum components into this element, plus the force $F_{i}$ applied on the element. This yields

$$
\left(\int_{w} \rho v_{i} \mathrm{~d} z\right)^{\bullet} \mathrm{d} x \mathrm{~d} y=-\left(\int_{w}\left(\rho v_{i}\right) v_{k} \mathrm{~d} z\right)_{, k} \mathrm{~d} x \mathrm{~d} y+F_{i}
$$

in which

$$
\begin{aligned}
F_{i} & =-(p w)_{, i} \mathrm{~d} x \mathrm{~d} y+p w_{, i} \mathrm{~d} x \mathrm{~d} y+2 \tau_{i 3} \mathrm{~d} x \mathrm{~d} y+\rho w g_{i} \mathrm{~d} x \mathrm{~d} y \\
& =\left(-w p_{, i}+2 \tau_{i 3}+\rho w g_{i}\right) \mathrm{d} x \mathrm{~d} y
\end{aligned}
$$

where $p=$ pressure, considered constant throughout the thickness, $g_{i}=$ gravity acceleration components, $\tau_{i 3}=$ shear stress due to viscosity of water (Figure 2), $p w=$ resultant of $p$ over the cross-section and $p w_{, i}=$ in-plane component of the normal force applied on crack surfaces inclined at angles $w_{, i}$.

At high flow rates, in-plane shear stresses due to viscosity $\mu$ may become important. Because the in-plane shear rate is $\dot{\gamma}_{12}=\left(v_{1,2}+v_{2,1}\right)$, and the normal deviatoric strain rates at negligible $\partial w / \partial t$ are $\dot{e}_{11}=\left(2 v_{1,1}-v_{2,2}\right) / 3$ and $\dot{e}_{22}=\left(2 v_{2,2}-v_{1,1}\right) / 3$, the deviatoric in-plane viscous stresses are $s_{12}=\mu \dot{\gamma}_{12}, s_{11}=2 \mu \dot{e}_{11}$ and $s_{22}=2 \mu \dot{e}_{22}$. Thus, the associated averaged net force $\left(w \bar{s}_{i k}\right)_{, k} \mathrm{~d} x \mathrm{~d} y$ has to be added to the term - $w p_{, i} \mathrm{~d} x \mathrm{~d} y$ in equation (4). Normally, however, this term is negligible compared with the effect of $\tau_{x z}$ and $\tau_{y z}$.

For the case of laminar flow (low Reynolds number), the velocity profile is parabolic, i.e., $v_{i}=v_{i}^{0}\left(1-4 z^{2} / w^{2}\right)$ with $v_{i}^{0}=3 \bar{v}_{i} / 2$. Since $\tau_{i 3}=\mu\left(\partial v_{i} / \partial z\right)$ at $z=w / 2$, we have $2 \tau_{i 3}=$ $-12 \mu \bar{v}_{i} / w=-12 \mu q_{i} / \rho w^{2}$. Assuming further that the profiles of $v_{1}$ and $v_{2}$ are both the same as the profile of $|v|$, we further have $\int_{w} \rho v_{i} v_{k} \mathrm{~d} z=\alpha \rho w \bar{v}_{i} \bar{v}_{k}$, in which

$$
\alpha=\frac{1}{\left(\bar{v}_{x}\right)^{2} w} \int_{w} v_{x}^{2} \mathrm{~d} z
$$

For a parabolic profile, $\alpha=1 \cdot 2$. 
Upon substitution of all expressions into equations (4) and (3), the balance of linear momentum is expressed as

$$
\dot{q}_{i}+\alpha\left(\frac{1}{\rho w} q_{i} q_{k}\right)_{, k}+\frac{12 \mu}{\rho w^{2}} q_{i}+w p_{, i}-\rho w g_{i}=\left(w \bar{s}_{i k}\right)_{, k} \quad(i=1,2)
$$

Note that the extended Bernoulli equation is a special case of this equation; the last term corresponds to the hydrostatic head, the fourth term to the pressure head, the second term to the velocity head and the third term is the head loss due to viscous friction.

\section{Balance of energy}

The total energy is the sum of the internal energy $U$ and the kinetic energy $K$. The flux of $U$ consists of energy convection by moving water and of heat conduction along the crack. The former flux is $\int_{w} \rho U v_{i} \mathrm{~d} z$ or $U q_{i}$. Thus, the rate of flow of internal energy into the element $\mathrm{d} x \mathrm{~d} y$ is $-\left(U q_{k}\right)_{, k} \mathrm{~d} x \mathrm{~d} y$. The flux due to heat conduction is $-k_{\mathrm{w}} w T_{, i}$,where $k_{w}=$ heat conductivity of water, $T=$ temperature and the corresponding rate of heat flow into element $\mathrm{d} x \mathrm{dy}$ is $\left(k_{\mathrm{w}} w T_{, k}\right)_{, k} \mathrm{~d} x \mathrm{~d} y$. The rate of heat flow into this element from the surrounding rock on both sides of the crack is $2 h\left(T_{\mathrm{r}}-T\right) \mathrm{d} x \mathrm{~d} y$, where $T_{\mathrm{r}}=$ remote rock temperature and $h=$ effective heat transfer coefficient. The flux of kinetic energy for the whole cross-section is $\int_{w}\left(\frac{1}{2} \rho v_{i} v_{i}\right) v_{k} \mathrm{~d} z$ which may be written as $\left(\beta \rho w \bar{v}_{i} \bar{v}_{i}\right) \bar{v}_{k}$ or $\left(q_{i} q_{i}\right) q_{k} \beta /(\rho w)^{2}$, in which

$$
\beta=\frac{1}{\left(\bar{v}_{x}\right)^{3} w} \int_{w} \frac{1}{2} v_{x}^{3} \mathrm{~d} z
$$

For a parabolic velocity profile, $\beta=27 / 35=0.771$. The rate of flow of kinetic energy into element $\mathrm{d} x \mathrm{~d} y$ is $-\left[\left(q_{i} q_{i}\right) q_{k} \beta /(\rho w)^{2}\right]_{, k} \mathrm{~d} x \mathrm{~d} y$.

The rate of work done by the stresses on the cross-sections forming the sides of the element $\mathrm{d} x \mathrm{~d} y$ is $-p \int_{w} v_{i} \mathrm{~d} z=-p w v_{i}=-p q_{k} / \rho$ per unit area. The net rate of all work done on all four sides of the element is $-\left(p q_{k} / \rho\right)_{, k} \mathrm{~d} x \mathrm{~d} y$. Furthermore, the rate of work of the reaction that acts on the crack surface, $p \dot{w} \mathrm{~d} x \mathrm{~d} y$, must also be included.

The kinetic energy in the whole cross-section is $\int_{w} \frac{1}{2} \rho v_{k} v_{k} \mathrm{~d} z$ or $(\alpha / 2 \rho w) q_{k} q_{k}$. Adding the internal energy $\rho w U$, the rate of change of all energy contained within element $\mathrm{d} x \mathrm{~d} y$ is $\left[\rho w U+q_{k} q_{k} \alpha /(2 \rho w)\right]^{*} \mathrm{~d} x \mathrm{~d} y$.

Collecting all terms, we have

$$
\left(\rho w U+\frac{\alpha}{2 \rho w} q_{k} q_{k}\right)^{\cdot}=2 h\left(T_{\mathrm{r}}-T\right)-\left(U q_{k}\right)_{, k}-\left(\frac{P}{\rho} q_{k}\right)_{, k}-p \dot{w}-\left[\frac{\beta}{(\rho w)^{2}}\left(q_{i} q_{i}\right) q_{k}\right]_{, k}+\left(k_{\mathrm{w}} w T_{, k}\right)_{, k}
$$

It is interesting to observe that the term $(\rho w U)^{\circ}+\left(U q_{k}\right)_{, k}$ appearing in equation (8) represents the material time derivative $D(\rho w U) / D t$ based on averaged velocity $\bar{v}_{i}$. Furthermore, the terms $\left(p q_{k} / \rho\right)_{, k}+p \dot{w}$ can be expressed by means of the averaged material time derivative as $\rho w D(p / \rho) / D t-\dot{p} w$.

\section{Properties of water and rock}

Variables $p, T$ and $\rho$ are related through the equation of state of water, $\rho=\rho(p, T)$. In a differential form, $\mathrm{d} \rho / \rho=\mathrm{d} p / K_{\mathrm{w}}-\alpha_{\mathrm{w}} \mathrm{d} T$, in which $K_{\mathrm{w}}=\rho /(\partial \rho / \partial p)=$ bulk modulus of water and $\alpha_{w}=-(\partial \rho / \partial T) / \rho=$ thermal dilatation coefficient. Furthermore, the heat capacity at constant volume may be calculated as $C_{\mathrm{v}}=T(\partial S / \partial T)_{\rho}$. In the numerical solution of the problem, the equations of state $\rho=\rho(p, T)$ and $S=S(p, T)$ have been used in the form of approximate mathematical expressions given in Reference 12, and a special subroutine for the properties of 
water has been programmed. In calculations it has been assumed that within each cross-section there exists only one phase of water, which practically has always been the liquid state (i.e., cavitation has been disallowed in advance). The viscosity of liquid water has been assumed according to the approximate formula $\mu=0 \cdot 279 /(T-5 \cdot 8) \mathrm{g}(\mathrm{cm} \mathrm{s})^{-1}, T$ being given in ${ }^{\circ} \mathrm{C}^{10}$

\section{Quasi-steady water circulation}

Consider now the problem of geothermal heat extraction from hot dry rock. Forcing water into a borehole, typically 3 or $4 \mathrm{~km}$ deep, a large crack is created in the rock mass by hydraulic fracturing (Figure 1). Because the horizontal (tectonic) stress is usually less than the vertical stress, the crack would normally be vertical and its shape may be assumed roughly as circular, of a typical diameter 1 to $1.5 \mathrm{~km}$. This crack is then intersected in its upper portion by a second borehole, and forced circulation of water through the crack is then begun. Soon a quasi-steady state is established and subsequent changes are very slow; they are governed primarily by the cooling of the rock, which has a two-fold effect: a smaller rate of heat transfer to the crack, and a greater opening width of the crack due to rock contraction. The width of the crack, however, always remains very small compared with the crack size, and so the terms involving $w_{, k}$ may be omitted. Moreover, the energy term due to the work of stresses, i.e., $\left(p q_{k} / \rho\right)_{, k}+p \dot{w}$, is small compared to the energy change due to the heating of water. Owing to the relatively small rate of flow of water in the narrow crack, the effect of kinetic energy on the heat balance may be also neglected, and so may be the in-plane deviator stresses due to viscosity. Heat conduction in water is negligible, too. Thus, the heat transfer is governed by convection due to the movement of water.

Under the conditions just mentioned, equations (1), (6) and (8) for a quasi-steady state reduce to the form

Mass balance:

$$
q_{k, k}=0 \quad(k=1,2)
$$

Momentum balance:

$$
q_{i}=-\frac{\rho w^{2}}{12 \mu}\left(w p, i-\rho w g_{i}-f_{i}\right), \quad \text { with } f_{i}=-\left(\frac{\alpha}{\rho w} q_{i}\right)_{, k} q_{k}
$$

Energy balance:

$$
q_{k} U_{, k}-2 \bar{h}\left(T_{\mathrm{r}}-T\right)=0
$$

in which equation (9) has been used to simplify the expression for $f_{i}$ and the first term of equation (10).

The derivative of internal energy $U$ in equation (11) may be evaluated from the relation $\mathrm{d} U=T \mathrm{~d} S-p \mathrm{~d} V$, where $S=$ entropy per unit mass, $V=1 / \rho, \mathrm{d} S=(\partial S / \partial T)_{V} \mathrm{~d} T+$ $(\partial S / \partial V)_{\mathrm{T}} \mathrm{d} V,(\partial S / \partial T)_{\mathrm{V}}=C_{\mathrm{v}} / T$ and $(\partial S / \partial V)_{\mathrm{T}}=(\partial p / \partial T)_{\mathrm{V}}=\alpha_{\mathrm{w}} / K$. Thus,

$$
\mathrm{d} U=T\left(\frac{C_{\mathrm{v}}}{T} \mathrm{~d} T+\frac{\alpha_{\mathrm{w}}}{K} \mathrm{~d} V\right)-p \mathrm{~d} V
$$

or

$$
U_{, k}=\frac{\partial U}{\partial T} T_{, k}+\frac{\partial U}{\partial V} V_{, k}=C_{v} T_{, k}+\left(p-\frac{\alpha_{w}}{K} T\right) \frac{1}{\rho^{2}} \rho_{, k}
$$

The last term in this equation is small, and so the approximation $U_{, k} \approx C_{\mathrm{v}} T_{, k}$ has been used in practical calculations. 
Equations (9), (10) and (11) represent a system of three differential equations for $p, q_{i}$ and $T$. Equations (9) and (10) are basically of diffusion type, which can be seen if the quadratic term $f_{i}$ is neglected and $q_{i}$ is substituted in equation (9). Equation (11) essentially governs temperature. Quadratic terms such as $q_{i}, q_{k}$ and $q_{k} T$, as well as the strong dependence of $w, \rho$, $U$ and $\mu$ upon $p$ and $T$, make the equation system highly non-linear.

Certain terms in the foregoing equations are small in most practical situations and may be neglected, as will be indicated in the following. Nevertheless, to be able to check the magnitude of the neglected effects, it is useful to have a rather complete formulation as given herein.

The rate of heat flow from the rock into the crack is determined by the temperature gradient at a given time. Because the depth of penetration $\delta$ of cooling into the rock is generally much less than the length of the crack, the heat conduction in the rock can be treated as essentially one-dimensional, in the direction $z$ normal to the crack. Furthermore, the time changes of temperature in the crack are relatively slow, and so the heat conduction may be approximated by assuming that the water temperature $T(t)$ has been essentially constant up to the current time. Considering the temperature profile in the direction normal to the crack as approximately parabolic, the penetration depth of cooling (thickness of thermal boundary layer) is obtained as $\delta=\left(12 t k_{\mathrm{r}} / \rho_{\mathrm{r}} C_{\mathrm{r}}\right)^{1 / 2}$ in which $k_{\mathrm{r}}=$ heat conductivity of the rock, $C_{\mathrm{r}}=$ its heat capacity and $\rho_{\mathrm{r}}=$ its mass density. For a parabola, the temperature gradient in the rock near the surface of the crack is $2\left(T_{\mathrm{r}}-T\right) / \delta$, where $T_{\mathrm{r}}=$ remote rock temperature (beyond depth $\delta$ ) and the heat flux into the crack from both sides is roughly $2\left[2 k_{\mathrm{r}}\left(T_{\mathrm{r}}-T\right) / \delta\right]$. Equating this to the expression $2 \bar{h}\left(T_{\mathrm{r}}-T\right)$ from equation (8), we obtain

$$
\bar{h} \approx \frac{2 k_{\mathrm{r}}}{\delta} \approx \sqrt{ }\left(\frac{k_{\mathrm{r}} \rho_{\mathrm{r}} C_{\mathrm{r}}}{3 t}\right)
$$

In the derivation the surface rock temperature was assumed to equal water temperature.

To estimate the width of the crack, the major effect on the opening of the crack stems from thermal contraction of the rock due to cooling. Again, assuming a parabolic temperature profile in the rock, the thermal opening width is

$$
w_{\mathrm{T}} \approx \frac{2}{3} \frac{\alpha_{\mathrm{r}}}{1-\nu_{\mathrm{r}}}\left(T_{\mathrm{r}}-T\right) \delta \approx \frac{2 \alpha_{\mathrm{r}}}{3\left(1-\nu_{\mathrm{r}}\right)}\left(T_{\mathrm{r}}-T\right) \sqrt{\left(\frac{12 k_{\mathrm{r}}}{\rho_{\mathrm{r}} C_{\mathrm{r}}}\right)}
$$

in which $\alpha_{\mathrm{r}}\left(1-\nu_{\mathrm{r}}\right)=$ thermal dilatation coefficient of the rock in direction $z$ at zero strains $\varepsilon_{x x}$ and $\varepsilon_{y y}$ in $x$ - and $y$-directions, $\alpha_{\mathrm{r}}=$ coefficient of free thermal dilatation and $\nu_{\mathrm{r}}=$ Poisson's ratio of the rock.

An additional crack opening is caused by the part of pressure $p$ which is in excess of the tectonic stresses that existed before the crack was induced. For a uniform pressure $p$ within a penny-shaped crack in an infinite homogeneous isotropic medium the opening width of the pressurized crack may be approximately calculated, according to fracture mechanics: ${ }^{13}$

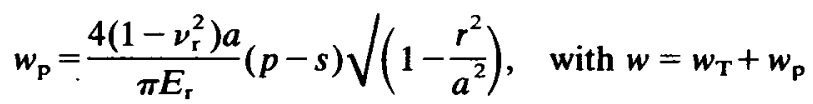

in which $E_{\mathrm{r}}=$ Young's modulus of the rock, $a=$ radius of the crack and $r=$ radial coordinate. Actually, however, pressure is rather non-uniform, and formula (15) would then give only very crude estimates. Therefore, $w_{\mathrm{p}}$ has been considered in the numerical calculations as fixed, assuming the radial opening profiles at $T-T_{\mathrm{r}}$ to be an ellipse of width $w=3 \mathrm{~mm}$ in the centre. $^{4}$ 


\section{FINITE ELEMENT SOLUTION}

\section{Variational method for pressure}

Intuitively, determination of pressures within the crack in rock is basically a diffusion-type problem, which suggests that equations (9) and (10) should be treated by finite elements similarly to all diffusion problems. In this light, it is natural to postulate the variational principle $\delta \Pi_{1}=0$ in which

$$
\Pi_{1}=\int_{\Omega} \frac{\rho w^{3}}{12 \mu}\left(\frac{1}{2} p_{, k}-\rho w g_{k}-f_{k}\right) p_{, k} \mathrm{~d} x \mathrm{~d} y-\int_{L} p q_{k} n_{k} \mathrm{~d} s \quad(k=1,2)
$$

Here $\Omega$ is the domain of the crack, $L$ is the boundary curve and $\left(n_{1}, n_{2}\right)$ is its unit outward normal. The field variable $p$ is assumed to satisfy boundary conditions $p=p_{\mathrm{b}}$ on that part of the boundary $\left(L_{\mathrm{p}}\right)$ where pressure $p_{\mathrm{b}}$ is prescribed. Variables $\rho, w, \mu$, the quadratic force terms $f_{1}, f_{2}$ (equation (10)) and fluxes $q_{k}$ at the boundary are to be treated as fixed when taking the variation, i.e.,

$$
\delta \Pi_{1}=\int_{\Omega} \frac{\rho w^{3}}{12 \mu}\left(p_{, k}-\rho w g_{k}-f_{k}\right) \delta p_{, k} \mathrm{~d} x \mathrm{~d} y-\int_{L} q_{k} \delta p n_{k} \mathrm{~d} s=0
$$

To prove that this implies the differential equations of the problem, Gauss's integral theorem may be applied, which provides

$$
\delta \Pi_{1}=-\int_{\Omega} \frac{\rho w^{3}}{12 \mu}\left[p_{, k k}-\left(\rho w g_{k}+f_{k}\right)_{, k}\right] \delta p \mathrm{~d} x \mathrm{~d} y+\int_{L}\left[\frac{\rho w^{3}}{12 \mu}\left(p_{, k}-\rho w g_{k}-f_{k}\right)+g_{k}\right] \delta p n_{k} \mathrm{~d} s=0
$$

If this is to be satisfied for any admissible function $p(x, y)$, the bracketed expressions must vanish, which yields equation (10) for the boundary points and equation (9) in which equation (10) is substituted as the field equation. The natural boundary conditions implied by equation (17) are either a prescribed pressure or a prescribed flux component normal to the contour of the crack.

\section{Variational method for heat convection}

The heat convection problem may be approximately treated as if the water flux $q_{k}$ in equation (11) were fixed. This equation, along with $U_{, k}=C_{\mathrm{v}} T_{, k}$ (equation (12)), involves no higher than first derivatives of temperature $T$, and so a suitable variational functional may be obtained by the least-square residual method, minimizing the functional

$$
\Pi_{2}=\int_{\Omega}\left(L T^{\prime}-F\right)^{2} \mathrm{~d} x \mathrm{~d} y, \quad \text { with } L T^{\prime}=q_{k} C_{\mathrm{v}} T_{, k}^{\prime}+2 \bar{h} T^{\prime}, F=2 \bar{h}\left(T_{\mathrm{r}}-T_{\mathrm{b}}\right)
$$

in which $T^{\prime}=T-T_{\mathrm{b}}$ and $T_{\mathrm{b}}=$ prescribed constant boundary temperature. Obviously; the condition $\delta \Pi_{2}=0$ yields equation (11) (with $U_{, k}=C_{v} T_{, k}$ ), along with the boundary conditions of prescribed temperature. Again, $C_{\mathrm{v}}$ and $q_{k}$ must be treated as fixed when taking the variation.

As an alternative, a Galerkin-type functional has been also considered, but equation (19) seemed to be more effective. 


\section{Discretization and numerical solution}

For the sake of simplicity, triangular finite elements with linear distribution functions for $p$ and $T$ have been adopted. Other variables, such as $w, q_{i}, C_{\mathrm{v}}, \rho$ and $\mu$, are assumed to be constant within each triangle; they are evaluated on the basis of $p$ and $T$ values interpolated for the centroid of the triangle and the derivatives of $p$ which are constant according to the distribution functions. The distribution function for pressure is introduced into $\delta \Pi_{1}=0$ (equation (17)), which yields a system of algebraic equations that are linear in the nodal values of $p$. The distribution function for temperature is introduced into $\delta \Pi_{2}=0$, which yields a system of algebraic equations that are linear in the nodal values of $T$. The equation matrix of the whole system is assembled in the usual manner from the elemental matrices, for which explicit expressions have been derived. The coefficients of the equation system depend on $p$ and $T$, which makes the problem non-linear. An iterative procedure has been adopted to solve this system of equations; the coefficients are first evaluated for assumed values of $p$ and $T$, and the system of linear equations is solved, yielding new values of $p$ and $T$. Using these new values of $p$ and $T$, the values of the coefficients are corrected and the system of linear equations is solved again; this is repeated many times. Experience indicated that the iterations converged reasonably well.

\section{NUMERICAL RESULTS AND DISCUSSION}

To check the finite element computer program for the steady state, various test cases where an approximate solution can be obtained by other means have been run. These included (a) parallel flow through a rectangular crack, in which a flux of hot water is prescribed on one side and the opposite side is at zero pressure and (b) flow in the same crack, but from a concentrated source on one side.

An idealized typical situation envisaged for the tentative geothermal hot dry rock scheme (essentially the same problem as that considered in Reference 4) has then been analysed. The penny-shaped vertical crack has a diameter of $1,000 \mathrm{~m}$. The water inlet is on the vertical line of symmetry, $150 \mathrm{~m}$ below the centre of the crack, and the water outlet is also on this line of symmetry, $150 \mathrm{~m}$ below the top of the crack. ${ }^{4}$ The initial rock temperature, $T_{\mathrm{r}}$, is assumed to vary linearly with depth and be $200^{\circ} \mathrm{C}$ at the top of the crack and $300^{\circ} \mathrm{C}$ at the bottom of the crack. The outlet pressure, which must be initially at least equal to the original tectonic stress, is considered to be $30 \mathrm{MN} / \mathrm{m}^{2}$ at all times. The boundary condition at the inlet is assumed to correspond to a constant pumping rate, giving a prescribed source of $150 \mathrm{~kg}$ water $/ \mathrm{s}$, of temperature $65^{\circ} \mathrm{C}$. The rock properties (granite) are assumed as $\rho=2,650 \mathrm{~kg} / \mathrm{m}^{3}, 8 \times$ $10^{-6} /{ }^{\circ} \mathrm{C}, v_{\mathrm{r}}=0.305, E_{\mathrm{r}}=37,600 \mathrm{MN} / \mathrm{m}^{2}$; the heat capacity is taken as approximately a constant, $\quad C_{\mathrm{v}}=95.4 \mathrm{~J}\left(\mathrm{~kg}^{\circ} \mathrm{C}\right)^{-1}$ while the viscosity has been indicated before. The thermal conductivity of the rock was considered as $k_{\mathrm{r}}=5.44 \mathrm{~J}\left(\mathrm{~ms}^{\circ} \mathrm{C}\right)^{-1}$, although $2.72 \mathrm{~J}\left(\mathrm{~ms}^{\circ} \mathrm{C}\right)^{-1}$ may have been more appropriate for a granite.

Using the iterative finite element program developed, the fields of water flux vector, temperature and crack width have been calculated for four different times $t$ after the start of pumping; see Figure 3 showing the fields for one half of the crack. The finite element subdivision is indicated by the location of the origins of the flux vectors in Figure 3. The results are qualitatively similar to those obtained by the finite difference method in Reference 4 .

In the flux vector fields, it is noteworthy that an eddy current develops and strongly intensifies, with the progress of cooling. This improves the effectiveness of heat extraction because water molecules travel a longer path in the hot rock. Also, note that at the inlet the flux is not directed toward the outlet, but goes away from the outlet, toward the bottom of the 

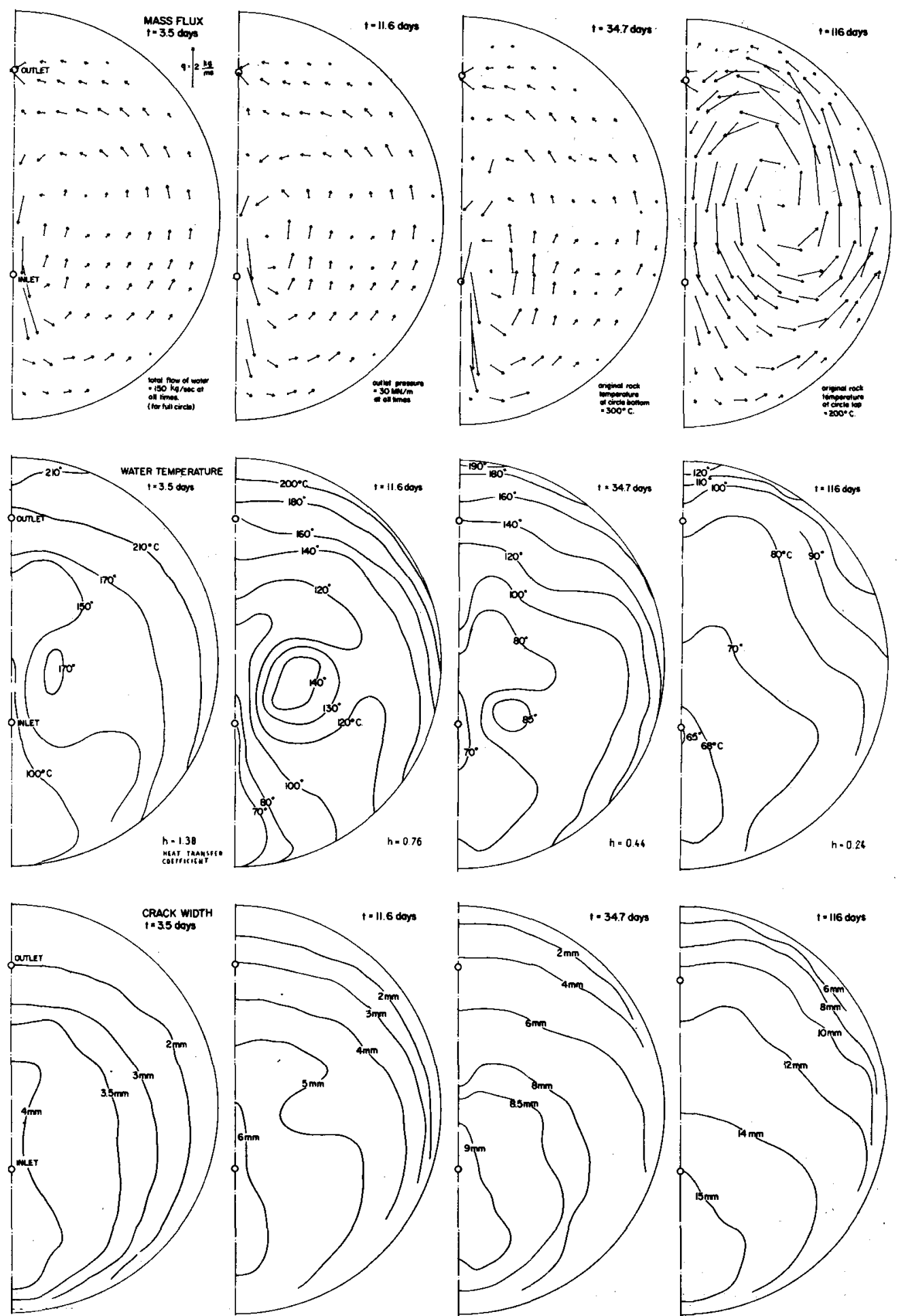

Figure 3. Calculated fields of water flux vector, temperature and crack width at various times after the start of circulation 
crack. This phenomenon, which has been found before in a different type of analysis, ${ }^{4}$ is very favourable for this geothermal scheme, for it allows best cooling where the rock is hottest, which would tend to extend the rock by further thermal fracturing (omitted here) in the hottest rock. ${ }^{1,2}$ The main reason for the downward flow at the inlet is no doubt buoyancy or gravity, as colder water is heavier. The increasing magnitude of the water fluxes and of the intensity of eddy currents is caused largely by the wider opening of the crack at later times.

From the temperature fields, it is apparent that the outlet temperature falls rapidly with time, and after about 1 month of pumping it drops below a level which would be usable for power generation. This means, of course, that the situation analysed is not feasible as a geothermal energy scheme. The rapid drop in outlet temperature is caused by the great decrease in the heat transfer rate from uncooled rock toward the crack surfaces as the penetration depth of cooling, $\delta$, increases.

The only chance of making the scheme workable is a secondary fracturing of the rock due to cooling, as has been pointed out before. ${ }^{1,2}$ The cooling of rock is definitely sufficient to cause cracks. These secondary cooling cracks would propagate from the crack walls orthogonally to the vertical plane of the main crack that was created by hydraulic fracturing. An important question is the opening width of the secondary cracks, which would determine whether a significant portion of the water flux from the main crack would divert into the secondary cracks, and whether eddy currents helping the heat convection would form within the secondary cracks. If this indeed happens, heat would be withdrawn from the tips of the secondary cracks, where the heat transmission is effective, and the rock at the crack tips would be cooled effectively, causing further extension of the secondary cracks. In this manner, a self-driving system of secondary cooling cracks might be envisaged.

Whether or not a significant water circulation could take place in the secondary cracks will be determined by the opening width $w_{2}$. At a given temperature drop in the rock, $w_{2}$ is roughly proportional to the crack spacing $l$. Assuming laminar flow, the water flux and the heat convection rate would be roughly proportional to $w_{2}^{3}$ or $l^{3}$ per crack, and the overall heat withdrawal rate would be proportional to $l^{2}{ }^{2}$.

This indicates that the spacing of the cooling cracks is of paramount importance for the feasibility of the geothermal energy scheme. A separate study has been devoted to this problem, ${ }^{14}$ considering first an idealized planar situation of a half plane cooled only by heat conduction through the rock, the rock surface being held at a fixed temperature. The problem is theoretically rather interesting; it represents a stability problem in fracture mechanics, and the solution does not depend only on crack tip energy release rates, but also on their partial derivatives with regard to crack lengths. It was found that in this case every other cooling crack would close when the ratio of crack depth to crack spacing reaches roughly $1 \cdot 7$, which would seem to be favourable for the geothermal scheme. However, it would be necessary to analyse crack spacing and direction under more realistic assumptions, especially including (a) water circulation (with eddy formation) and heat convection by water in the secondary cracks, (b) the effect of heat convection in the secondary cracks upon the opening width of the main crack, (c) the three-dimensional nature of the pattern of secondary cracks, (d) secondary cooling cracks at the bottom edge of the main crack, as distinct from those in the middle of the walls of the main crack and (e) extensions and closures of the main crack, non-circular shapes of the crack, and other effects.

Although the iterations of the finite element solution appeared to converge well, it has not been possible to investigate the magnitude of the error. Since the problem is highly non-linear, the results must, therefore, be regarded with caution and should not be expected to be very accurate. 


\section{CONCLUSION}

Water circulation, heat transfer and opening width in a large crack in hot dry non-porous rock mass can be effectively solved by finite elements. The results are favourable for some aspects of the proposed geothermal heat extraction scheme. However, further important questions remain to be solved.

\section{ACKNOWLEDGEMENT}

The work has been supported by the U.S. Energy Research and Development Administration under Grant No. AER75-00187 and was carried out at Northwestern University while the second author was on leave from the University of Tokyo during the 1975-76 academic year. Thanks are due to Professor S. Nemat-Nasser of Northwestern University who, as co-principal investigator and co-director of the project, made many useful critical comments and suggestions while he worked in parallel on the same problem. Furthermore, Professor T. Mura of Northwestern University and H. Abé, Visiting Scholar, are thanked for making one rather valuable suggestion.

\section{REFERENCES}

1. M. Smith, H. Potter, D. Brown and R. L. Aamodt, 'Induction and growth of fractures in hot rock', in Geothermal Energy, P. Kruger and C. Otte (Eds), pp. 251-268, Stanford University Press, Stanford, California, 1973.

2. M. C. Smith, R. L. Aamodi, R. M. Potter and D. W. Brown, Proc. 2nd Geothermal Energy Symp., San Francisco, Cal. (1975)

3. F. G. West, 'Dry hot rock project', in New Mexico Geological Society Guidebook, pp. 355-358, 25th Field Conf., Ghost Ranch, N.M., 1974.

4. R. D. McFarland, 'Geothermal reservoir models-Crack plane model', Report LA-5947-MS, UC-13, Los Alamos Scientific Laboratory (1975).

5. Z. P. Bažant, S. Nemat-Nasser and H. Ohtsubo, 'Finite element solution of geothermal energy extraction', NSF Workshop on Geothermal Reservoir Engineering, P. Krueger and H. J. Ramey (Eds), pp. 232-239, Stanford University, 1975.

6. S. Nemat-Nasser and Z. P. Bažant, 'Basic equations for fluid flow and heat transfer through hydraulically induced fractions in hot dry rock masses', Progress Report on NSF-RANN Grant No. AER-00187, Dept. of Civil Engineering, Northwestern University (1976).

7. J. W. Mercer and G. F. Pinder, 'Finite element analysis of hydrothermal systems', manuscript privately communicated to Z. P. Bažant (1975).

8. J. W. Mercer, G. F. Pinder and I. G. Donaldson, 'A Galerkin finite element analysis of the hydrothermal system at Wairakei, New Zealand', J. Geophys. Res. 80, 2608-2621 (1975).

9. J. W. Mercer and C. R. Faust, 'Simulation of water- and vapor-dominated hydrothermal reservoirs', Paper SPE 5520, Society of Petroleum Engineers of AIME, New York (1975).

10. F. H. Harlow and W. Pracht, 'A theoretical study of geothermal energy extraction', J. Geophys. Res. 77, 7083-7048 (1972).

11. J. R. Welty, C. E. Wicks and R. E. Wilson, Fundamentals of Momentum, Heat and Mass Transfer, Wiley, New York, 1969.

12. 'Thermodynamic and transport properties of steam', in ASTM Steam Tables, 2nd edition, American Society of Mechanical Engineers, New York, 1967.

13. I. N. Sneddon and M. Lowengrub, Crack Problems in the Classical Theory of Elasticity, Wiley Interscience, 1969, Eq. 3.3.11.

14. Z. P. Bažant and H. Ohtsubo, 'Stability conditions for propagation of a system of cracks in a brittle solid', Mech. Res. Comnuun. 4, 353-366 (1977). 